\title{
Vortex dynamics in nonrelativistic version of Abelian Higgs model: Effects of the medium on the vortex motion
}

\author{
Arkadii Kozhevnikov ${ }^{1,2, \star}$ \\ ${ }^{1}$ Laboratory of Theoretical Physics, S. L. Sobolev Institute for Mathematics \\ ${ }^{2}$ Novosibirsk State University
}

\begin{abstract}
The closed vortex dynamics is considered in the nonrelativistic version of the Abelian Higgs Model. The effect of the exchange of excitations propagating in the medium on the vortex string motion is taken into account. The obtained are the effective action and the equation of motion both including the exchange of the propagating excitations between the distant segments of the vortex and the possibility of its interaction with the static fermion asymmetric background. They are applied to the derivation of the time dependence of the basic geometrical contour characteristics.
\end{abstract}

\section{Introduction}

The string-like (or vortex) solutions of the field-theoretical models are widely discussed in application to numerous physical systems, from the really observed in condensed matter physics (quantized vortices in $\mathrm{He}^{4}$ [1], Abrikosov lines in type II superconductors [2], vortices in Bose - Einstein condensates [3] to hypothetical cosmic strings [4]. Of particular interest are the situations when the dynamics of the vortex contours can be deduced from the field equations of the underlying field theory. The dynamics of cosmic strings was studied in the framework of the Nambu-Goto action [5] which was shown to result from the relativistic version of the Abelian Higgs model (AHM) [6]. Transverse motions of the Nambu-Goto string are typically with the velocity of light [5]. The solution in the form of the static cosmic string was obtained from the static version of AHM [7]. Another limiting case is the nonrelativistic one, which can be derived from the nonrelativistic Abelian Higgs model (NRAHM). The static limit of this model coincides with the Ginzburg-Landau theory [8]. Another interesting aspect of the time-dependent gauge vortex solutions is that they can elucidate the interrelations among such characteristics of the closed contours as the helicity of gauge field forming the vortex, the writhe and torsion (twist) numbers and their possible dependence on time [9-11].

The aim of the present talk is to consider the dynamical evolution of the closed gauge vortex string in NRAHM, including the possibility of its interaction with the static fermion asymmetric background. The corresponding equation of motion is obtained, side by side with expressions for the time derivatives of the curvature and torsion of the gauge vortex contour [12-14].

^e-mail: kozhev@math.nsc.ru 


\section{Nonrelativistic Abelian Higgs Model with the gauge vortex}

Nonrelativistic Abelian Higgs model incorporating gauge vortices is given by the Lagrangian density

$$
\begin{aligned}
\mathcal{L}= & \frac{1}{8 \pi}\left(\boldsymbol{E}^{2}-\boldsymbol{H}^{2}\right)-\frac{g}{2}\left(|\psi|^{2}-n_{0}\right)^{2}+\frac{1}{2}\left[\psi^{*}\left(i \hbar \partial_{t}-q \varphi+q a_{0}\right) \psi+\text { c.c. }\right]- \\
& \frac{1}{2 m}\left|\left(-i \hbar \nabla-\frac{q}{c} \boldsymbol{A}+\frac{q}{c} \boldsymbol{a}\right) \psi\right|^{2}-\rho_{0} \varphi
\end{aligned}
$$

The source of the vortex with the contour $\boldsymbol{X}(\sigma, t)$ is the singular phase of the Higgs field $\chi_{s}, a_{\mu}=$ $-\frac{\hbar c}{q} \partial_{\mu} \chi_{s}$ :

$$
[\boldsymbol{\nabla} \times \boldsymbol{\nabla}] \chi_{\mathrm{s}}=2 \pi \oint d \sigma \boldsymbol{X}^{\prime} \delta^{(3)}(\boldsymbol{x}-\boldsymbol{X}(\sigma, t))
$$

The solution of Eq. (2) is

$$
\begin{aligned}
a_{0} & =\frac{\hbar}{2 q} \oint \frac{\left(\left[\dot{\boldsymbol{X}} \times \boldsymbol{X}^{\prime}\right], \boldsymbol{x}-\boldsymbol{X}\right)}{|\boldsymbol{x}-\boldsymbol{X}|^{3}} d \sigma \\
\boldsymbol{a} & =\frac{\hbar c}{2 q} \oint \frac{\left[\boldsymbol{X}^{\prime} \times(\boldsymbol{x}-\boldsymbol{X})\right]}{|\boldsymbol{x}-\boldsymbol{X}|^{3}} d \sigma .
\end{aligned}
$$

In what follows, all fields are represented as the sum of the background fields and the fluctuations. As usual, the background fields are found from the condition that the terms in the action which are linear in the fluctuations, should vanish while the terms quadratic in the latter will give the quantum corrections to the effective action. The expressions for the background fields are [12]:

$$
\begin{aligned}
\boldsymbol{A}(\boldsymbol{x}, t) & =\int \frac{d^{3} k}{(2 \pi)^{3}} \frac{i \Phi_{0} / \lambda_{L}^{2}}{\boldsymbol{k}^{2}\left(\boldsymbol{k}^{2}+1 / \lambda_{L}^{2}\right)} \times \oint d \sigma\left[\boldsymbol{k} \times \boldsymbol{X}^{\prime}\right] e^{i(\boldsymbol{k}, \boldsymbol{x}-\boldsymbol{X})} \\
\boldsymbol{H}(\boldsymbol{x}, t) & =\int \frac{d^{3} k}{(2 \pi)^{3}} \frac{\Phi_{0} / \lambda_{L}^{2}}{\boldsymbol{k}^{2}+1 / \lambda_{L}^{2}} \times \oint d \sigma \boldsymbol{X}^{\prime} e^{i(\boldsymbol{k}, \boldsymbol{x}-\boldsymbol{X})} \\
\boldsymbol{E}(\boldsymbol{x}, t) & =-\int \frac{d^{3} k}{(2 \pi)^{3}} \frac{\Phi_{0} /\left(c \lambda_{L}^{2}\right)}{\boldsymbol{k}^{2}+1 / \lambda_{L}^{2}} \oint d \sigma e^{-i(\boldsymbol{k}, \boldsymbol{x}-\boldsymbol{X})}\left\{\left[\dot{\boldsymbol{X}} \times \boldsymbol{X}^{\prime}\right]-\frac{\boldsymbol{k}\left(\boldsymbol{k}\left[\dot{\boldsymbol{X}} \times \boldsymbol{X}^{\prime}\right]\right)}{\boldsymbol{k}^{2}}\right\}
\end{aligned}
$$

where $\Phi_{0}=\frac{2 \pi \hbar c}{q}, \lambda_{L}=\sqrt{\frac{m c^{2}}{4 \pi n_{0} q^{2}}}$, and the constrain $\rho_{0}+q n_{0}=0$ provides the net charge neutrality. The modulus of the Higgs field is assumed to be uniform except the vortex lines where it tends to zero.

When evaluating the action of the background fields,

$$
S_{\mathrm{bg}}=\int d^{4} x\left[\frac{1}{8 \pi}\left(\boldsymbol{E}^{2}-\boldsymbol{H}^{2}\right)-\frac{1}{8 \pi \lambda_{L}^{2}}(\boldsymbol{A}-\boldsymbol{a})^{2}+n_{0} q a_{0}\right],
$$

it is useful to represent it in the form $S_{\mathrm{bg}}=S_{\mathrm{bg}}^{(0)}+\Delta S_{\mathrm{bg}}$, where

$$
\begin{aligned}
S_{\mathrm{bg}}^{(0)}= & \frac{\Phi_{0}^{2}}{8 \pi} \int d t \frac{d^{3} k}{(2 \pi)^{3}}\left(\frac{1 / \lambda_{L}^{2}}{\mathbf{k}^{2}+1 / \lambda_{L}^{2}}\right)^{2} \oint d \sigma_{1} d \sigma_{2} e^{-i \boldsymbol{k} \boldsymbol{X}_{12}} \times \\
& \left\{\left[\dot{\boldsymbol{X}}_{1} \times \boldsymbol{X}_{1}^{\prime}\right] \cdot\left[\dot{\boldsymbol{X}}_{2} \times \boldsymbol{X}_{2}^{\prime}\right] / c^{2}-\left(\boldsymbol{X}_{1}^{\prime} \boldsymbol{X}_{2}^{\prime}\right)\left(1+\lambda_{L}^{2} \boldsymbol{k}^{2}\right)\right\}+n_{0} q \int d^{4} x a_{0}= \\
& \frac{\Phi_{0}^{2}}{32 \pi^{2} \lambda_{L}^{2}} \int d t d \sigma_{1} d \sigma_{2} e^{-\left|\boldsymbol{X}_{21}\right| / \lambda_{L}}\left\{\frac{\left[\dot{\boldsymbol{X}}_{1} \times \boldsymbol{X}_{1}^{\prime}\right]\left[\dot{\boldsymbol{X}}_{2} \times \boldsymbol{X}_{2}^{\prime}\right]}{2 c^{2} \lambda_{L}}-\frac{\left(\boldsymbol{X}_{1}^{\prime} \boldsymbol{X}_{2}^{\prime}\right)}{\left|\boldsymbol{X}_{21}\right|}\right\}+n_{0} q \int d^{4} x a_{0}
\end{aligned}
$$


is the infrared finite contribution while

$$
\begin{aligned}
\Delta S_{\mathrm{bg}}= & -\frac{\Phi_{0}^{2}}{8 \pi c^{2}} \int d t \int \frac{d^{3} k}{(2 \pi)^{3} \boldsymbol{k}^{2}}\left(\frac{1 / \lambda_{L}^{2}}{\mathbf{k}^{2}+1 / \lambda_{L}^{2}}\right)^{2} \times \\
& \oint d \sigma_{1} d \sigma_{2} e^{-i \boldsymbol{k} \boldsymbol{X}_{12}}\left(\boldsymbol{k}\left[\dot{\boldsymbol{X}}_{1} \times \boldsymbol{X}_{1}^{\prime}\right]\right)\left(\boldsymbol{k}\left[\dot{\boldsymbol{X}}_{2} \times \boldsymbol{X}_{2}^{\prime}\right]\right)
\end{aligned}
$$

represents the infrared divergent contribution. Hereafter the notation are: $\boldsymbol{X}_{1,2} \equiv \boldsymbol{X}\left(\sigma_{1,2}\right)$, dot and prime stand for the derivatives over time and the corresponding contour parameter $\sigma_{1,2}$, respectively.

Using $\psi=n^{1 / 2} e^{i \chi}$, the fields are represented in terms of the background fields and fluctuations: $\varphi \rightarrow \varphi+\delta \varphi, \boldsymbol{A} \rightarrow \boldsymbol{A}+\delta \boldsymbol{A}, n \rightarrow n_{0}+\delta n, \chi \rightarrow \delta \chi$, where $\delta n$ and $\delta \chi$ are the Higgs mode and Goldstone mode, respectively. The action of fluctuations is

$$
S_{\mathrm{f}}^{(0)}=\int d^{4} x\left\{\frac{1}{2} f^{T} M f-\frac{1}{8 \pi} \delta \boldsymbol{A}\left(-\frac{1}{c^{2}} \partial_{t}^{2}+\boldsymbol{\nabla}^{2}-\frac{1}{\lambda_{L}^{2}}\right) \delta \boldsymbol{A}+q a_{0} \delta n\right\}+\ldots .
$$

where $f^{T}=(\delta n, \delta \chi, \delta \varphi)$, and .... represents the derivatives $\partial^{3} \boldsymbol{X} / \partial t^{3}$ or higher which can be neglected in nonrelativistic case [12]. The matrix $M$ looks like

$$
M=\left(\begin{array}{ccc}
\frac{\hbar^{2}}{4 m n_{0}} \boldsymbol{\nabla}^{2}-g & -\hbar \partial_{t} & -q \\
\hbar \partial_{t} & \frac{\hbar^{2} n_{0}}{m} \nabla^{2} & 0 \\
-q & 0 & -\frac{1}{4 \pi} \nabla^{2}
\end{array}\right),
$$

which permits one to obtain the propagator matrix in the four-momentum representation:

$$
M^{-1}=\frac{1}{\left(\frac{\epsilon_{B}^{2}}{\hbar^{2}}+\frac{c^{2}}{\lambda_{L}^{2}}-\omega^{2}-i 0\right)}\left(\begin{array}{ccc}
-\frac{n_{0}}{m} \boldsymbol{k}^{2} & -\frac{i \omega}{\hbar} & -\frac{4 \pi n_{0} q}{m} \\
\frac{i \omega}{\hbar} & -\frac{m}{\hbar^{2} \boldsymbol{k}^{2} n_{0}}\left(\frac{\epsilon_{B}^{2}}{\hbar^{2}}+\frac{c^{2}}{\lambda_{L}^{2}}\right) & \frac{4 \pi i \omega q}{\hbar \boldsymbol{k}^{2}} \\
-\frac{4 \pi n_{0} q}{m} & -\frac{4 \pi i \omega q}{\hbar \boldsymbol{k}^{2}} & \frac{4 \pi}{\boldsymbol{k}^{2}}\left(\frac{\epsilon_{B}^{2}}{\hbar^{2}}-\omega^{2}\right)
\end{array}\right)
$$

where $\epsilon_{B}^{2} \equiv \epsilon_{B}^{2}(\boldsymbol{k})=\left(\frac{\hbar^{2} \boldsymbol{k}^{2}}{2 m}\right)^{2}+\hbar^{2} c_{s}^{2} \boldsymbol{k}^{2}$ is the square of the Bogolyubov spectrum [15], $c_{s}=\sqrt{\frac{n_{0} g}{m}}$ is the sound velocity. The freely propagating excitations obey the equations of motion:

$$
\begin{aligned}
0 & =\hbar \partial_{t} \delta \chi+g \delta n+q \delta \varphi-\frac{\hbar^{2}}{4 m n_{0}} \nabla^{2} \delta n, \\
0 & =\partial_{t} \delta n+\frac{\hbar n_{0}}{m} \nabla^{2} \delta \chi .
\end{aligned}
$$

Upon excluding $\delta \chi$ one obtains

$$
\left(-\partial_{t}^{2}+c_{s}^{2} \nabla^{2}-\frac{c^{2}}{\lambda_{L}^{2}}-\frac{\hbar^{2}}{4 m^{2}} \nabla^{4}\right) \delta n=0
$$

resulting in the above dispersion law. The static limit with $q=0$ gives $\nabla^{2} \delta n-\xi^{-2} \delta n=0$, where $\xi=\hbar / 2 m c_{s}$ is the correlation length which, by the order of magnitude, is the distance where the Higgs field goes to zero near the string core. Formally, $\xi$ is the ultraviolet cutoff reflecting the ignorance of the Higgs field profile at short distances.

The dominant quantum correction to the action due to exchange of excitations propagating in the medium is

$$
\Delta S_{\mathrm{f}}=\frac{1}{2} \int \frac{d^{4} k}{(2 \pi)^{4}}\left(\frac{\epsilon_{B}^{2}}{\hbar^{2}}+\frac{c^{2}}{\lambda_{L}^{2}}-\omega^{2}-i 0\right)^{-1} \frac{n_{0} q^{2}}{m}\left|a_{0 k}\right|^{2} \boldsymbol{k}^{2} .
$$


It is treated by using the derivative expansion for $I=\int_{-\infty}^{\infty} \frac{d \omega}{2 \pi} \cdot \frac{\left|f_{k}\right|^{2}}{\omega^{2}-\Omega_{k}^{2}+i 0}$ :

$$
\begin{aligned}
I= & \int_{-\infty}^{\infty} d t d \tau \frac{d \omega}{2 \pi} \frac{f_{\boldsymbol{k}}(t) f_{\boldsymbol{k}}^{*}(t+\tau) e^{i \omega \tau}}{\omega^{2}-\Omega_{\boldsymbol{k}}^{2}+i 0}=\int d t f_{\boldsymbol{k}}(t) \sum_{l=0}^{\infty} \frac{(-i)^{l}}{l !} \frac{d^{l} f_{\boldsymbol{k}}^{*}}{d t^{l}}\left[\frac{\partial^{l}}{\partial \omega^{l}} \frac{1}{\omega^{2}-\Omega_{\boldsymbol{k}}^{2}}\right]_{\omega=0}= \\
& \int d t\left[-\frac{\left|f_{\boldsymbol{k}}(t)\right|^{2}}{\Omega_{k}^{2}}-\frac{1}{2 \Omega_{k}^{4}}\left(f_{\boldsymbol{k}} \frac{\partial^{2} f_{\boldsymbol{k}}^{*}}{\partial t^{2}}+\text { c.c. }\right)+\cdots\right] .
\end{aligned}
$$

Then the leading correction to the effective action arising from the exchange of fluctuations can be represented in the form

$$
\Delta S_{\mathrm{f}}=\frac{\Phi_{0}^{2}}{8 \pi \lambda_{L}^{2}} \int d t d \sigma_{1} d \sigma_{2} \frac{d^{3} k}{(2 \pi)^{3}} \frac{e^{i \boldsymbol{k} \cdot \boldsymbol{X}_{21}}}{\frac{\hbar^{2} \boldsymbol{k}^{4}}{4 m^{2}}+c_{s}^{2} \boldsymbol{k}^{2}+\frac{c^{2}}{\lambda_{L}^{2}}} \frac{\left(\boldsymbol{k}\left[\dot{\boldsymbol{X}}_{1} \times \boldsymbol{X}_{1}^{\prime}\right]\right)\left(\boldsymbol{k}\left[\dot{\boldsymbol{X}}_{2} \times \boldsymbol{X}_{2}^{\prime}\right]\right)}{\boldsymbol{k}^{2}} .
$$

It has the finite part contributing to the effective action, and the infrared divergent piece cancelling analogous divergence in the background action Eq. (6). In fact, the sum of the infrared divergent contribution of the background action and that of due to the exchange of the Higgs mode represented in the form

$$
\begin{aligned}
\Delta S_{\mathrm{eff}}= & \frac{\Phi_{0}^{2}}{8 \pi c^{2}} \int d t d \sigma_{2} d \sigma_{2}\left[\dot{\boldsymbol{X}}_{1} \times \boldsymbol{X}_{1}^{\prime}\right]_{i}\left[\dot{\boldsymbol{X}}_{2} \times \boldsymbol{X}_{2}^{\prime}\right]_{j} \nabla_{21 i} \nabla_{21 j} I\left(\sigma_{1}, \sigma_{2}\right), \\
I\left(\sigma_{1}, \sigma_{2}\right)= & \frac{\partial}{\partial \lambda_{L}^{2}}\left[\lambda_{L}^{2} \int \frac{d^{3} k}{(2 \pi)^{3}}\left(\frac{1}{\boldsymbol{k}^{2}}-\frac{1}{\boldsymbol{k}^{2}+1 / \lambda_{L}^{2}}\right) e^{i \boldsymbol{k} \boldsymbol{X}_{21}}\right]- \\
& \int \frac{d^{3} k}{(2 \pi)^{3}} e^{i \boldsymbol{k} \boldsymbol{X}_{21}}\left[\frac{1}{\boldsymbol{k}^{2}}-\frac{\boldsymbol{k}^{2}+1 / \xi^{2}}{\boldsymbol{k}^{4}+\boldsymbol{k}^{2} / \xi^{2}+1 / \xi^{2} \lambda_{s}^{2}}\right],
\end{aligned}
$$

demonstrates explicitly the cancellation of the infrared divergences. Here, the new length parameter arises: $\lambda_{s}=\lambda_{L} \cdot \frac{c_{s}}{c} \ll \lambda_{L}$. In what follows, the assumption $\xi \ll \lambda_{s} \ll \lambda_{L}$ is adopted.

All expressions in the vortex action are of the type $\int d t \oint d \sigma_{1} d \sigma_{2} F\left(\sigma_{1}, \sigma_{2}\right) \exp ^{-\left|X_{21}\right| / \lambda_{L}}$, i.e. nonlocal. The expansion $\boldsymbol{X}_{2}=\boldsymbol{X}_{1}+z \boldsymbol{X}_{1}^{\prime}+\frac{z^{2}}{2} \boldsymbol{X}_{1}^{\prime \prime}+\frac{z^{3}}{6} \boldsymbol{X}_{1}^{\prime \prime \prime}+\cdots$ in $z=\sigma_{2}-\sigma_{1}$ admits the local limit. The local form of the action is

$$
S_{\mathrm{eff}} \approx\left(\frac{\Phi_{0}}{4 \pi \lambda_{L}}\right)^{2} \int d t d \sigma\left\{\frac{1}{2 c_{s}^{2}}\left[\dot{\boldsymbol{X}} \times \boldsymbol{X}^{\prime}\right]^{2} \ln \frac{\lambda_{s}}{\xi}-\boldsymbol{X}^{\prime 2} \ln \frac{\lambda_{L}}{\xi}\right\}+n_{0} q \int d^{4} x a_{0} .
$$

The leading kinetic term $\left[\dot{\boldsymbol{X}} \times \boldsymbol{X}^{\prime}\right]^{2} / c_{s}^{2}$ is due to the exchange of Higgs field excitations. It is strongly enhanced as compared with the bare one. The similar kinetic term in the background action is suppressed as $c_{s}^{2} / c^{2}$ and does not contain the logarithmic enhancement factor $\ln \lambda_{s} / \xi$.

\section{Equation of the vortex motion}

When obtaining the equations of motion the local limit should be taken after making the variations.

They are fulfilled by using the expressions $\delta \int d^{4} x a_{0}=-\frac{\Phi_{0}}{c} \int d t d \sigma\left(\delta \boldsymbol{X}\left[\dot{\boldsymbol{X}} \times \boldsymbol{X}^{\prime}\right]\right)$,

$$
\begin{aligned}
\delta I \equiv & \delta \oint d \sigma_{1} d \sigma_{2}\left(\boldsymbol{X}_{1}^{\prime} \boldsymbol{X}_{2}^{\prime}\right) \frac{e^{-\left|\boldsymbol{X}_{21}\right| / \lambda_{L}}}{\left|\boldsymbol{X}_{21}\right|}=2 \oint d \sigma_{1} d \sigma_{2}\left(\delta \boldsymbol{X}_{1} \cdot\left[\boldsymbol{X}_{1}^{\prime} \times\left[\boldsymbol{X}_{21} \times \boldsymbol{X}_{2}^{\prime}\right]\right]\right) \frac{1+\left|\boldsymbol{X}_{21}\right| / \lambda_{L}}{\left|\boldsymbol{X}_{21}\right|^{3}} \times \\
& e^{-\left|\boldsymbol{X}_{21}\right| / \lambda_{L}}=2 \oint d \sigma \int_{-\infty}^{\infty} d z \frac{e^{-|z| / \lambda_{L}}}{2|z|} \delta \boldsymbol{X} \cdot\left[\boldsymbol{X}^{\prime} \times\left[\boldsymbol{X}^{\prime} \times \boldsymbol{X}^{\prime \prime}\right]\right]=-2 \ln \frac{\lambda_{L}}{\xi} \oint d \sigma \delta \boldsymbol{X} \cdot \boldsymbol{X}^{\prime \prime}
\end{aligned}
$$


and analogous (cumbersome) expressions for the variation of the terms containing the time derivatives. The variation of the effective action is

$$
\begin{aligned}
\delta S_{\mathrm{eff}}= & -\left(\frac{\Phi_{0}}{4 \pi \lambda_{L}}\right)^{2} \int d t d \sigma \delta \boldsymbol{X} \cdot\left\{\frac{1}{c_{s}^{2}}\left[\boldsymbol{X}^{\prime} \times \frac{\partial}{\partial t}\left[\dot{\boldsymbol{X}} \times \boldsymbol{X}^{\prime}\right]\right] \ln \frac{\lambda_{s}}{\xi}-\boldsymbol{X}^{\prime \prime} \ln \frac{\lambda_{L}}{\xi}\right\}- \\
& \frac{n_{0} q \Phi_{0}}{c} \int d t d \sigma\left(\delta \boldsymbol{X}\left[\dot{\boldsymbol{X}} \times \boldsymbol{X}^{\prime}\right]\right),
\end{aligned}
$$

resulting in the equation of the vortex motion

$$
\left[\dot{\boldsymbol{X}} \times \boldsymbol{X}^{\prime}\right]=\frac{\hbar}{2 m}\left(\boldsymbol{X}^{\prime \prime} \ln \frac{\lambda_{L}}{\xi}+\frac{1}{c_{s}^{2}}\left[\frac{\partial}{\partial t}\left[\dot{\boldsymbol{X}} \times \boldsymbol{X}^{\prime}\right] \times \boldsymbol{X}^{\prime}\right] \ln \frac{\lambda_{s}}{\xi}\right) .
$$

When the exchange of excitation is turning off, $c_{s} \rightarrow \infty$, the solution of Eq. (9) is the ring vortex moving in the direction perpendicular to its plane with velocity [12]

$$
\dot{\boldsymbol{X}}=\boldsymbol{b} \frac{\hbar}{2 m}\left|\boldsymbol{X}^{\prime \prime}\right| \ln \frac{\lambda_{L}}{\xi} .
$$

One can include the static background of chiral fermions (with asymmetry characterized by the chemical potential $\mu_{f}$ ) coupled to the gauge field $A_{\mu}$. This results in the term $-\mu_{f} N_{\mathrm{CS}}$ [16-20], where

$$
N_{\mathrm{CS}}=\frac{1}{\Phi_{0}^{2}} \int d^{3} x(\boldsymbol{A},[\boldsymbol{\nabla} \times \boldsymbol{A}])=\frac{h_{A}}{\Phi_{0}^{2}}
$$

is the Abelian Chern-Simons number related with helicity

$$
\begin{aligned}
h_{A}= & i \Phi_{0}^{2} \oint d \sigma_{1} d \sigma_{2} \int \frac{d^{3} k}{(2 \pi)^{3} \boldsymbol{k}^{2}}\left(\frac{1 / \lambda_{L}^{2}}{\boldsymbol{k}^{2}+1 / \lambda_{L}^{2}}\right)^{2} \times \\
& \left(\boldsymbol{k},\left[\frac{\partial \boldsymbol{X}\left(\sigma_{1}\right)}{\partial \sigma_{1}} \times \frac{\partial \boldsymbol{X}\left(\sigma_{2}\right)}{\partial \sigma_{2}}\right]\right) \exp \left[-i\left(\boldsymbol{k}, \boldsymbol{X}\left(\sigma_{1}\right)-\boldsymbol{X}\left(\sigma_{2}\right)\right] .\right.
\end{aligned}
$$

The variation of the Chern-Simons induced contribution to the equation of the vortex motion is deduced from the variation of helicity:

$$
\begin{aligned}
\delta h_{A}= & 2 \Phi_{0}^{2} \int \frac{d^{3} k}{(2 \pi)^{3}}\left(\frac{1 / \lambda_{L}^{2}}{\boldsymbol{k}^{2}+1 / \lambda_{L}^{2}}\right)^{2} \oint d \sigma_{1} d \sigma_{2}\left(\delta \boldsymbol{X}_{1},\left[\boldsymbol{X}_{1}^{\prime} \times \boldsymbol{X}_{2}^{\prime}\right]\right) e^{-i\left(\boldsymbol{k}, \boldsymbol{X}_{12}\right)}= \\
& \frac{\Phi_{0}^{2}}{4 \pi \lambda_{L}^{3}} \oint d \sigma_{1} d \sigma_{2}\left(\delta \boldsymbol{X}_{1},\left[\boldsymbol{X}_{1}^{\prime} \times \boldsymbol{X}_{2}^{\prime}\right]\right) e^{-\left|\boldsymbol{X}_{12}\right| / \lambda_{L}} \\
= & \frac{\Phi_{0}^{2}}{8 \pi \lambda_{L}^{3}} \oint d \sigma\left(\left[\boldsymbol{X}^{\prime} \times \boldsymbol{X}^{\prime \prime \prime}\right], \delta \boldsymbol{X}\right) \int_{-\infty}^{\infty} z^{2} e^{-|z| / \lambda_{L}} d z=\frac{\Phi_{0}^{2}}{2 \pi} \oint d \sigma\left(\left[\boldsymbol{X}^{\prime} \times \boldsymbol{X}^{\prime \prime \prime}\right], \delta \boldsymbol{X}\right) .
\end{aligned}
$$

Taking all the above considerations into account one obtains the equation of motion which, in the gauge $\left(\dot{\boldsymbol{X}}, \boldsymbol{X}^{\prime}\right)=0$, has the following form:

$$
\frac{1}{c_{0}^{2}} \ddot{\boldsymbol{X}}-\boldsymbol{X}^{\prime \prime}+\frac{1}{\gamma}\left[\dot{\boldsymbol{X}} \times \boldsymbol{X}^{\prime}\right]+\frac{\tilde{\mu}}{\gamma}\left[\boldsymbol{X}^{\prime} \times \boldsymbol{X}^{\prime \prime \prime}\right]=0
$$


where

$$
\begin{aligned}
\gamma & =\frac{\hbar}{2 m} \ln \frac{\lambda_{L}}{\xi}, \\
T_{0} & =\frac{\hbar}{2 m c_{s}^{2}} \ln \frac{\lambda_{s}}{\xi}, \\
\tilde{\mu} & =\frac{\mu_{f}}{4 \pi^{2} \hbar n_{0}}, \\
c_{0} & =\left(\frac{\gamma}{T_{0}}\right)^{1 / 2}=c_{s}\left(\frac{\ln \lambda_{L} / \xi}{\ln \lambda_{s} / \xi}\right)^{1 / 2} .
\end{aligned}
$$

It looks like the wave equation added with the two additional terms. The term $\propto\left[\dot{\boldsymbol{X}} \times \boldsymbol{X}^{\prime}\right]$ is the analog of the Magnus force acting on the moving vortex due to the nonzero circulation of the supercurrent. The term $\propto\left[\boldsymbol{X}^{\prime} \times \boldsymbol{X}^{\prime \prime \prime}\right]$ is induced by the anomalous current $\boldsymbol{j}_{\text {an }} \propto \mu_{f} \boldsymbol{H}$ widely discussed in connection with chiral magnetic effect [21]. This current is subjected to the action of the Lorentz force $\propto\left[\boldsymbol{j}_{\text {an }} \times \boldsymbol{H}\right]$ :

$$
\boldsymbol{f}_{L} \propto \mu_{f} \int_{-\infty}^{\infty} d z\left[\boldsymbol{X}^{\prime}(\sigma) \times \boldsymbol{X}^{\prime}(\sigma+z)\right] e^{-|z| / \lambda_{L}} \propto \mu_{f}\left[\boldsymbol{X}^{\prime} \times \boldsymbol{X}^{\prime \prime \prime}\right]
$$

The static solution is helix with the constant curvature $\kappa=$ const and the torsion related with the amount of fermion asymmetry $\tau=\gamma / \tilde{\mu}$ [13]. See the next section for the definition of these geometric quantities.

\section{Time derivatives of the basic contour characteristics}

The basic contour vectors of normal $\boldsymbol{n}$, bi-normal $\boldsymbol{b}$, and tangent $\boldsymbol{X}^{\prime}$ constitute the right triple, $\boldsymbol{X}^{\prime}=$ $[\boldsymbol{n} \times \boldsymbol{b}]$ and obey the Frenet-Serret equations:

$$
\frac{\partial}{\partial \sigma}\left(\begin{array}{c}
\boldsymbol{X}^{\prime} \\
\boldsymbol{n} \\
\boldsymbol{b}
\end{array}\right)=\left(\begin{array}{ccc}
0 & \kappa & 0 \\
-\kappa & 0 & \tau \\
0 & -\tau & 0
\end{array}\right)\left(\begin{array}{c}
\boldsymbol{X}^{\prime} \\
\boldsymbol{n} \\
\boldsymbol{b}
\end{array}\right)
$$

They are of unit length. Here, $\kappa$ and $\tau$ are the curvature and the torsion, respectively. One can write the equations determining the time derivatives of the triple $\left(\boldsymbol{n}, \boldsymbol{b}, \boldsymbol{X}^{\prime}\right)$ using the fact that they are the unit orthogonal vectors:

$$
\frac{\partial}{\partial t}\left(\begin{array}{c}
\boldsymbol{X}^{\prime} \\
\boldsymbol{n} \\
\boldsymbol{b}
\end{array}\right)=\left(\begin{array}{ccc}
0 & a_{X n} & a_{X b} \\
a_{n X} & 0 & a_{n b} \\
a_{b X} & a_{b n} & 0
\end{array}\right)\left(\begin{array}{c}
\boldsymbol{X}^{\prime} \\
\boldsymbol{n} \\
\boldsymbol{b}
\end{array}\right) \equiv \hat{A}\left(\begin{array}{c}
\boldsymbol{X}^{\prime} \\
\boldsymbol{n} \\
\boldsymbol{b}
\end{array}\right)
$$

Using the relation the commutativity of derivatives $\partial_{t} \partial_{\sigma}-\partial_{\sigma} \partial_{t}=0$ one can further restrict the matrix $\hat{A}$ :

$$
\hat{A}=\left(\begin{array}{ccc}
0 & a_{X n} & a_{X b} \\
-a_{X n} & 0 & a_{n b} \\
-a_{X b} & -a_{n b} & 0
\end{array}\right)
$$

and obtain the expressions

$$
\begin{aligned}
a_{n b} & =\left(\tau a_{X n}+a_{X b}^{\prime}\right) / \kappa, \\
\dot{\kappa} & =a_{X n}^{\prime}-\tau a_{X b}, \\
\dot{\tau} & =\kappa a_{X b}+a_{n b}^{\prime} .
\end{aligned}
$$


Introducing the vector $\boldsymbol{W}=\left[\dot{\boldsymbol{X}} \times \boldsymbol{X}^{\prime}\right]=W_{n} \boldsymbol{n}+W_{b} \boldsymbol{b}, \dot{\boldsymbol{X}}=W_{n} \boldsymbol{b}-W_{b} \boldsymbol{n}$ one gets $\frac{\partial \boldsymbol{X}^{\prime}}{\partial t}=\boldsymbol{b}\left(W_{n}^{\prime}-\tau W_{b}\right)-$ $\boldsymbol{n}\left(W_{b}^{\prime}+\tau W_{n}\right)$ which permits one to identify

$$
\begin{aligned}
& a_{X n}=-W_{b}^{\prime}-\tau W_{n}, \\
& a_{X b}=W_{n}^{\prime}-\tau W_{b} .
\end{aligned}
$$

Using the above relations the time derivatives of the curvature and torsion can be obtained:

$$
\begin{aligned}
\dot{\kappa} & =-\left(W_{b}^{\prime}+\tau W_{n}\right)^{\prime}-\tau\left(W_{n}^{\prime}-\tau W_{b}\right), \\
\dot{\tau} & =\kappa\left(W_{n}^{\prime}-\tau W_{b}\right)+\left\{\left[\left(W_{n}^{\prime}-\tau W_{b}\right)^{\prime}-\tau\left(W_{b}^{\prime}+\tau W_{n}\right)\right] / \kappa\right\}^{\prime} .
\end{aligned}
$$

The system of equations is closed by adding the equations of motion

$$
\begin{aligned}
W_{n}= & \kappa(\gamma-\tilde{\mu} \tau)+T_{0}\left\{\dot{W}_{b}+\left[\left(W_{n}^{\prime}-\tau W_{b}\right)^{\prime}-\tau\left(W_{b}^{\prime}+\tau W_{n}\right)\right] \times\right. \\
& \left.W_{n} / \kappa\right\}, \\
W_{b}= & \tilde{\mu} \kappa^{\prime}-T_{0}\left\{\dot{W}_{n}-\left[\left(W_{n}^{\prime}-\tau W_{b}\right)^{\prime}-\tau\left(W_{b}^{\prime}+\tau W_{n}\right)\right] W_{b} / \kappa\right\} .
\end{aligned}
$$

The interesting particular case is one with $T_{0}=0$ and $\tilde{\mu}=0$ corresponding to the neglect of the exchange of excitations and the fermion asymmetry. Then, after re-scaling $\kappa \rightarrow \gamma^{-1 / 2} \kappa, \tau \rightarrow \gamma^{-1 / 2} \tau$, $\sigma \rightarrow \gamma^{1 / 2} \sigma$ one gets

$$
\begin{aligned}
\dot{\kappa} & =-\left(2 \kappa^{\prime} \tau+\kappa \tau^{\prime}\right), \\
\dot{\tau} & =\left[\kappa \kappa^{\prime}+\left(\frac{\kappa^{\prime \prime}}{\kappa}-\tau^{2}\right)^{\prime}\right] .
\end{aligned}
$$

These are the Da-Rios-Betchov equations [22] first proposed in hydrodynamics of ideal fluid in 1906.

The equations (14) and (15) are strongly nonlinear, hence one needs some approximations to get consequences from them. We choose the solution exact in $\mu_{f}$ and of the first order in $T_{0}$. The derivation is straightforward, but the expressions are still cumbersome. Instead, one can find such useful interesting quantity as the time derivative of the total torsion:

$$
\oint \dot{\tau} d \sigma=\frac{\hbar \mu_{f}}{64 \pi^{2} m^{2} c_{s}^{2} n_{0}} \times \ln \frac{\lambda_{L}}{\xi} \times \ln \frac{\lambda_{s}}{\xi} \oint \kappa^{4} \tau^{\prime} d \sigma
$$

The total torsion is related with the twist:

$$
\mathrm{Tw}=\frac{1}{2 \pi} \oint \tau d \sigma=\frac{1}{2 \pi} \oint\left(\boldsymbol{X}^{\prime},\left[\boldsymbol{n}^{\prime} \times \boldsymbol{n}\right]\right) d \sigma .
$$

On the other hand, the time derivative of helicity is

$$
\begin{aligned}
\dot{h}_{A}= & \frac{\Phi_{0}^{2}}{2 \pi} \oint d \sigma\left(\left[\dot{\boldsymbol{X}} \times \boldsymbol{X}^{\prime}\right], \boldsymbol{X}^{\prime \prime \prime}\right)=\frac{\Phi_{0}^{2}}{2 \pi} \oint d \sigma\left(\boldsymbol{W}, \kappa^{\prime} \boldsymbol{n}+\kappa \tau \boldsymbol{b}\right)= \\
& \frac{\Phi_{0}^{2}}{2 \pi} \oint d \sigma\left(\kappa^{\prime} W_{n}+\kappa \tau W_{b}\right)=-\frac{\Phi_{0}^{2}}{2 \pi} \oint \dot{\tau} d \sigma \equiv-\Phi_{0}^{2} \frac{d \mathrm{Tw}}{d t} .
\end{aligned}
$$

The writhe number and its variation are, respectively,

$$
\mathrm{Wr}=\frac{1}{4 \pi} \oint d \sigma_{1} \oint d \sigma_{2} \frac{\left(\boldsymbol{X}_{12},\left[\boldsymbol{X}_{1}^{\prime} \times \boldsymbol{X}_{2}^{\prime}\right]\right)}{\left|\boldsymbol{X}_{12}\right|^{3}}=-i \int \frac{d^{3} k}{(2 \pi)^{3}} \oint d \sigma_{1} d \sigma_{2} \frac{\left(\boldsymbol{k},\left[\boldsymbol{X}_{2}^{\prime} \times \boldsymbol{X}_{1}^{\prime}\right]\right)}{\boldsymbol{k}^{2}} \times e^{i \boldsymbol{k} \cdot \boldsymbol{X}_{21}}
$$


and

$$
\begin{aligned}
\delta \mathrm{Wr}= & -i \int \frac{d^{3} k}{(2 \pi)^{3}} \frac{\boldsymbol{k}}{\boldsymbol{k}^{2}} \oint d \sigma_{1} d \sigma_{2}\left\{\left[\delta \boldsymbol{X}_{2}^{\prime} \times \boldsymbol{X}_{1}^{\prime}\right]+\left[\boldsymbol{X}_{2}^{\prime} \times \delta \boldsymbol{X}_{1}^{\prime}\right]+i\left[\boldsymbol{X}_{2}^{\prime} \times \boldsymbol{X}_{1}^{\prime}\right]\left(\boldsymbol{k} \cdot \boldsymbol{X}_{21}\right)\right\} e^{i \boldsymbol{k} \cdot \boldsymbol{X}_{21}}= \\
& \int \frac{d^{3} k}{(2 \pi)^{3}} \oint d \sigma_{1} d \sigma_{2}\left(\delta \boldsymbol{X}_{21} \cdot\left[\boldsymbol{X}_{2}^{\prime} \times \boldsymbol{X}_{1}^{\prime}\right]\right) e^{i \boldsymbol{k} \cdot \boldsymbol{X}_{21}}= \\
& \oint d \sigma_{1} d \sigma_{2}\left(\delta \boldsymbol{X}_{21} \cdot\left[\boldsymbol{X}_{2}^{\prime} \times \boldsymbol{X}_{1}^{\prime}\right]\right) \delta^{(3)}\left(\boldsymbol{X}_{21}\right) .
\end{aligned}
$$

The ambiguity $0 \times \infty$ in Eq. (17) is regularized with the help of expression

$$
\delta^{(3)}(\boldsymbol{X})=\lim _{\xi \rightarrow 0} \frac{e^{-\boldsymbol{X}^{2} / 2 \xi^{2}}}{(2 \pi)^{3 / 2} \xi^{3}},
$$

so that the regularized variation of the writhe becomes

$$
\begin{aligned}
\delta \mathrm{Wr}_{\text {reg }}= & \lim _{\xi \rightarrow 0} \frac{1}{(2 \pi)^{3 / 2} \xi^{3}} \oint d \sigma_{1} d \sigma_{2} e^{-\boldsymbol{X}_{21}^{2} / 2 \xi^{2}}\left(\delta \boldsymbol{X}_{21},\left[\boldsymbol{X}_{2}^{\prime} \times \boldsymbol{X}_{1}^{\prime}\right]\right)= \\
& \lim _{\xi \rightarrow 0} \frac{1}{(2 \pi)^{3 / 2} 2 \xi^{3}} \oint d \sigma \int_{-\infty}^{\infty} d z z^{2} e^{-z^{2} / 2 \xi^{2}} \times\left(\delta \boldsymbol{X},\left[\boldsymbol{X}^{\prime} \times \boldsymbol{X}^{\prime \prime \prime}\right]\right)= \\
& \frac{1}{2 \pi} \oint d \sigma\left(\delta \boldsymbol{X},\left[\boldsymbol{X}^{\prime} \times \boldsymbol{X}^{\prime \prime \prime}\right]\right)=\frac{\delta h_{A}}{\Phi_{0}^{2}} .
\end{aligned}
$$

The time derivative of writhe,

$$
\frac{d \mathrm{Wr}}{d t}=\frac{\dot{h}_{A}}{\Phi_{0}^{2}},
$$

permits one to establish the "conservation law":

$$
\frac{d}{d t}(\mathrm{Wr}+\mathrm{Tw})=0
$$

The sum $\mathrm{Wr}+\mathrm{Tw}$ is known from topology as the topological invariant called self-linking [23, 24]. It is obtained here as the consequence of the dynamical equations govern the gauge vortex motion.

\section{Discussion and conclusion}

We have shown that the vortex string motion admits the non-relativistic regime if one takes into account the effects of the medium resulting in the exchange of the excitations between the different segments of the string. However, the characteristic velocity of the transverse motion $c_{0}$ in the last line of Eq. (12) exceeds the velocity of sound. This may result in the Vavilov-Cherenkov damping. We leave this problem for a separate work. The kinetic term becomes strongly modified due to the exchange of fluctuations. The ring vortex motion due to the term $\left[\dot{\boldsymbol{X}} \times \boldsymbol{X}^{\prime}\right]$ in the medium consisting of the charged scalar field condensate is possible. The interaction with the fermion asymmetric background results in additional term $\left[\boldsymbol{X}^{\prime} \times \boldsymbol{X}^{\prime \prime \prime}\right]$ in equation of motion because of the anomalous current $\boldsymbol{j}_{\text {an }} \propto \mu_{f} \boldsymbol{B}$. The anomaly is the quantum effect hence the appearance of the Planck constant in the equation for such macroscopic quantity as the time derivative of the total twist, Eq. (16). Interesting enough, but the topological conservation of the sum $\mathrm{Tw}+\mathrm{Wr}$ is shown here to result from the dynamics

of the field system. 
As for the application to physics, one can hardly hope to observe the closed or curved vortices in type II superconductors immersed into the static external magnetic fields. However, their production could be quite possible in the fast temperature quench [25]. See, for example, Ref. [26], where such a mechanism was considered in the framework of the 2D Abelian Higgs Model. Also, numerous simulations of the phase transitions in the early Universe point to a considerable portion of the closed cosmic strings [4]. The type of the model considered in the present work is one where the charged particles interacting with the gauge field, condense uniformly except for the vortex core. The total neutrality is provided by some static background of the opposite charge. The cosmic strings of such type are not excluded in the models of the dark (hidden) sector [27, 28]. However, the relation of the present work parameters $q, n_{0}$, and $g$ with ones from the models of the hidden sector, goes beyond the scope of this communication.

\section{References}

[1] R. J. Donnelly, Quantized Vortices in Helium II (Cambridge University Press, 1991).

[2] A. A. Abrikosov, ZhETF 32, 1442 (1957).

[3] K. W. Madison, F. Chavy, W. Wohlleben, and J. Dalibard, Phys. Rev. Lett. 84, 806 (2000).

[4] A. Vilenkin and E. P. S. Shellard, Cosmic Strings and other Topological Defects( Cambridge University Press, 1994).

[5] J. Scherk, Rev. Mod. Phys. 47, 123 (1975).

[6] D. Förster, Nucl. Phys. B81, (1974) 84.

[7] H. B. Nielsen and P. Olesen, Nucl. Phys. B61, 45 (1973).

[8] V. L. Ginzburg and L. D. Landau, ZhETF 20, 1064 (1950).

[9] H. K. Moffatt, J. Fluid Mech. 35, 117 (1969).

[10] M. A. Berger and G. B. Field, J. Fluid Mech. 147, 133 (1984).

[11] H. K. Moffatt and R. L. Ricca, Proc. R. Soc. London A439, 411 (1992).

[12] A. A. Kozhevnikov, Int. Journal of Mod. Phys. B24, 605 (2010).

[13] A. A. Kozhevnikov, Phys. Lett. B461, 256 (1999).

[14] A. A. Kozhevnikov, Phys. Lett. B750, 122 (2015).

[15] N. N. Bogolyubov, J. Phys. (USSR) 11, 23 (1947).

[16] A. N. Redlich and L. C. R. Wijewardhana, Phys. Rev. Lett. 54, 970 (1985).

[17] V. A. Rubakov and A. N. Tavkhelidze, Theor. Math. Phys. 65, 250 (1985); Phys. Lett. B165, 109 (1985).

[18] M. Joyce and M. E. Shaposhnikov, Phys. Rev. Lett. 79, 1193 (1997).

[19] M. Laine, JHEP 0510:056 (2005).

[20] V. B. Semikoz and J. W. F. Valle, JCAP 11, 048 (2011).

[21] D. E. Kharzeev, Progress in Particle and Nuclear Physics 75, 133 (2014).

[22] L. S. Da Rios, Rend. Circ. Mat. Palermo 22, 117 (1906).

[23] F. B. Fuller, Proc. Natl. Acad. Sci. USA 68, 815, (1971).

[24] W. F. Pohl, J. Math. Mech. 17, 975, (1968).

[25] W. H. Zurek, Phys. Rep. 276, 177 (1996).

[26] G. J. Stephens, Luís M. A. Bettencourt, and W. H. Zurek, Phys. Rev. Lett. 88, 137004 (2002).

[27] T. Vachaspati, Phys. Rev. D80, 063502 (2009).

[28] J. M. Hyde, A. J. Long, and T. Vachaspati, Phys. Rev. D89, 065031 (2014). 